\title{
Oclusão Percutânea dos Canais Arteriais com Prótese Amplatzer
}

\author{
Francisco Chamié ${ }^{1,2,3}$, Daniel Chamié ${ }^{4}$, Sérgio Ramos ${ }^{1,2}$
}

\begin{abstract}
RESUMO
Introdução: Vários dispositivos têm sido utilizados para o fechamento percutâneo dos canais arteriais, com graus variáveis de sucesso. Dentre eles, destaca-se o oclusor ductal Amplatzer (Amplatzer Duct Occluder ${ }^{\circledR}$ - ADO) que, pela sua facilidade de uso, segurança e eficiência, em praticamente todos os tipos de canais, tem sido largamente utilizado. Neste trabalho, apresentamos a experiência do nosso grupo com este dispositivo, discutindo suas características, aspectos técnicos do seu implante, bem como os resultados obtidos com o seu emprego. Método: De abril de 1999 até dezembro de 2006, 116 pacientes foram encaminhados para oclusão percutânea. Destes, 91 (64F:27M) foram selecionados para fechamento percutâneo utilizando a prótese Amplatzer. O implante foi realizado da maneira tradicional, já descrita em outros trabalhos, na maioria dos casos. Nos PCA de menor diâmetro, foi necessária a dilatação da extremidade pulmonar do canal com cateter-balão para angioplastia coronariana. Os casos foram todos selecionados por meio de ecocardiogramas transtorácicos (ETT) com mapeamento de fluxo a cores. O seguimento foi feito com ETT no dia seguinte ao procedimento e com 1, 3, 6 e 12 meses (m) após. Resultados: As idades variaram de 2 a 600 meses $(48,0 \pm 116,4$ meses $)$ e os pesos de 4 a $88 \mathrm{~kg}(17,0 \pm 19,1 \mathrm{~kg})$. Os menores diâmetros dos canais variaram entre 0,5 e $11 \mathrm{~mm}(3,0 \pm 1,8 \mathrm{~mm})$, sendo 25 menores do que $2 \mathrm{~mm}$. Os tipos morfológicos se distribuíram da seguinte maneira: tipo $\mathrm{A}=58(64 \%)$; tipo $\mathrm{C}=6(7 \%)$; tipo $\mathrm{D}=2(2 \%)$ e tipo $\mathrm{E}=25(27 \%)$, de acordo com a classificação de Krichenko. Não houve nenhum canal do tipo B na nossa casuística. Foram usadas ADO em 90 casos, distribuídas segundo os tamanhos, como se segue: $5-4$ em 4 casos (4\%); 6-4 em 34 casos (37\%); 8-6 em 33 (37\%); $10-8$ em 16 (18\%) e $12-10$ em 3 (3\%). Em uma paciente de 50 anos com canal amplo e hipertensão arterial pulmonar grave, foi utilizada, com sucesso, uma prótese de CIV muscular (Amplatzer Muscular VSD Occluder ${ }^{\circledR}$ - VSD MUSC) de $12 \mathrm{~mm}$. O implante foi possível em 90 casos. No outro, a rosca defeituosa de uma prótese ADO 8-6 causou
\end{abstract}

\section{SUMMARY}

\section{Transcatheter Closure of Persistent Ductus Arteriosus with Amplatzer Duct Occluder}

Background: Several devices have been used for transcatheter closure of Patent Ductus Arteriosus (PDA), with varying degrees of success. Among them, the Amplatzer Duct Occluder ${ }^{\mathrm{TM}}$ (ADO) is now widely used due to ease of use, safety and efficacy to close almost all types of PDAs. The authors present their experience with this device and discuss its features, technical aspects of the implant and results obtained. Methods: From April 1999 to December 2006, 116 patients were subsequently submitted to transcatheter occlusion. Out of those, 91 (64F:27M) were selected for occlusion with the ADO. Implants were performed as described elsewhere, in most cases. Pulmonary extremity was dilated with coronary balloon catheters in smallest ductuses. All cases were selected based on previous transthoracic echos with color flow mapping (TTE). Follow-up was carried out through immediate, $1 \mathrm{~m}, 3 \mathrm{~m}$, $6 \mathrm{~m}$ and $12 \mathrm{~m}$ TTE. Results: Ages ranged from 2 to 600 months $(87.0 \pm 116.4 \mathrm{mo})$ and weight from 4 to $88 \mathrm{~kg}(23.8 \pm 19.1 \mathrm{~kg})$. Mean PDA diameter was $3.0 \pm 1.8 \mathrm{~mm}$ (ranging 0.5 to $11 \mathrm{~mm}$ ). Morphology of PDA was assessed, according to Krichenko's classification, and was of type A in 58 patients (64\%), type $C$ in 6 patients $(7 \%)$, type D in 2 patients $(2 \%)$ and type E in 25 patients (27\%). No type B PDAs were found in our series. Devices were successfully implanted in 90 patients. Type of devices used were PDA occluder 5-4 in 4 cases $(4 \%), 6-4$ in 34 cases $(37 \%), 8-6$ in 33 cases $(36 \%)$, $10-8$ in 16 cases $(18 \%), 12-10$ in 3 cases $(3 \%)$. In a 50 yearold female patient with severe pulmonary hypertension, a $12 \mathrm{~mm}$ Muscular VSD Occluder device was successfully implanted. In only one patient the implant was not achieved because of defective screwing mechanism that caused inadvertent release of the device in the descending aorta. Defect was closed and the device retrieved surgically, with no further complications. There was total occlusion in all other cases, as of 1-month follow-up TTE. All ductuses remained

CARPE - Cardiologia Pediátrica e Fetal, RJ.

2 INTERCAT - Cardiologia Intervencionista, RJ.

3 Hospital dos Servidores do Estado - MS, RJ.

${ }^{4}$ Instituto Dante Pazzanese de Cardiologia, SP.

Correspondência: Francisco Chamié. Rua Dois de Dezembro, 38 Sala 807. Flamengo. Rio de Janeiro, RJ. CEP 22220-040.

Tel. (21) 2246-1629 - Fax. (21) 2556-6833

e-mail: fchamie@intercat.med.br / fchamie@pobox.com

Recebido em: 08/01/2007 - Aceito em: 25/01/2007 
seu destacamento prematuro na aorta descendente. O paciente foi encaminhado para cirurgia eletiva, onde teve a prótese resgatada e seu canal ligado sem problemas. Em todos os que tiveram o implante, houve fechamento completo, sem fluxo residual dentro do primeiro mês do procedimento, e se mantêm fechados desde então. Não houve complicações significativas relacionadas ao uso da prótese. Não houve óbitos relacionados ao procedimento. Conclusões: $\mathrm{O}$ fechamento dos canais arteriais com a prótese Amplatzer é um procedimento extremamente simples, seguro e com alto índice de sucesso. É nossa opinião que deva ser adotada como primeira opção para a oclusão de todos os canais. O procedimento é possível mesmo nos canais de pequeno diâmetro $(<2 \mathrm{~mm})$, que são indicados para fechamento percutâneo.

DESCRITORES: Persistência do conduto arterioso. Cateterismo cardíaco. Próteses e implantes. Cardiopatias congênitas.

0 fechamento percutâneo dos canais arteriais com diversos tipos de oclusor é uma alternativa já estabelecida para o tratamento cirúrgico e tem tido vasta aceitação como tratamento de escolha na maioria dos centros em todo o mundo'.

O uso do oclusor ductal Amplatzer (Amplatzer Duct Occluder - ADO) já está bem divulgado e tem seu emprego, em crianças e adultos, já descrito amplamente ${ }^{2-8}$.

Neste trabalho, apresentamos a experiência do grupo com essas próteses e preconizamos o seu uso mesmo para os canais pequenos, com menor diâmetro inferior a $2 \mathrm{~mm}$.

\section{MÉTODO}

\section{Desenho do estudo}

Este é um estudo retrospectivo, no qual foram revisados os registros de todos os pacientes portadores de canal arterial persistente (PCA) selecionados para fechamento percutâneo, entre abril de 1999 e dezembro de 2006.

\section{População de pacientes}

No período mencionado, todos os pacientes portadores de PCA foram consecutivamente submetidos a avaliação para oclusão percutânea por meio de ecocardiogramas transtorácicos (ETT) com mapeamento de fluxo a cores. Foram excluídos os pacientes menores de 3 meses de idade e aqueles que apresentavam algum outro defeito que necessitasse de cirurgia. Foram estudados 116 pacientes, dos quais 91 receberam o implante de uma prótese Amplatzer (Tabela 1).

Dois pacientes eram portadores de síndrome de Down. Um deles havia sido operado, previamente, de comunicação interatrial (CIA) e comunicação interventricular (CIV) ampla. Outro caso apresentava anel fibroso subaórtico; nos demais, o canal arterial era o único defeito apresentado. closed thereafter. No major complications or procedurerelated deaths were observed. Conclusions: The Amplatzer Duct Occluder has proven to be user-friendly, safe and effective for transcatheter PDA closure. Based on our own experience and that of others, we suggest that it should become the method of choice for percutaneous PDA occlusion in ductuses of suitable anatomy.

DESCRIPTORS: Ductus arteriosus, patent. Heart catheterization. Prostheses and implants. Heart defects, congenital.

Apenas dois pacientes apresentavam sintomas de falência cardíaca: uma menina de 2 meses, pesando $3,6 \mathrm{~kg}$ (JF), que se apresentava em franca insuficiência cardíaca, teve seu caso realizado em caráter de emergência. Outra paciente de 50 anos, com fibrilação atrial crônica, hipertensão arterial sistêmica e pulmonar grave, conseqüente a um canal de grandes dimensões, mantinha-se em insuficiência cardíaca classe funcional II da NYHA, em tratamento clínico. Os demais eram assintomáticos.

Outros três tinham hipertensão arterial pulmonar grave, quase em nível sistêmico, mas sem hiper-resistência vascular pulmonar.

\section{Técnica do procedimento}

Todos os procedimentos foram realizados sob anestesia geral, com intubação orotraqueal. $O$ acesso foi obtido por punção de veia e artéria femoral.

Foi realizada heparinização sistêmica nas doses de $100 \mathrm{UI} / \mathrm{kg}$ ou $5000 \mathrm{UI}$, nos adultos. Foi usada, também, cefalotina venosa nas doses de $50 \mathrm{mg} / \mathrm{kg}$ (máximo de $2 \mathrm{~g}$ ), na sala de exame e mais duas doses de cefalexina oral de $25 \mathrm{mg} / \mathrm{kg}$ (ou $500 \mathrm{mg}$ ), a cada 8 horas, no pós-procedimento imediato para profilaxia antimicrobiana.

Em todos os pacientes, foi realizado o cateterismo direito e esquerdo, com registro de pressões em artéria pulmonar e aorta. Não foi realizado o cálculo da relação de fluxos (Qp/Qs) na nossa rotina de oclusão, a não ser nos que apresentaram hipertensão arterial pulmonar significativa ( $\geq$ a $75 \%$ da sistêmica em 4 casos).

As angiografias foram obtidas com injeções na aorta descendente nas incidências lateral esquerda e oblíqua anterior direita para obter a adequada análise morfológica do canal arterial segundo os critérios de Krichenko et al. ${ }^{9}$, bem como a medida do seu menor diâmetro (Figura 1). Nos casos cujo menor diâmetro 
TABELA 1

Caracterização da população do estudo

\begin{tabular}{|c|c|c|c|c|c|c|c|c|}
\hline$N$ & Id & Sexo & Idade (meses) & Peso (kg) & PAP $(\mathrm{mmHg})$ & $\Phi(\mathrm{mm})$ & Tipo & Prótese \\
\hline 1 & ALCS & $\mathrm{F}$ & 9 & 10 & $42 / 24(36)$ & 7,6 & C & $10-8$ \\
\hline 2 & $\mathrm{LHC}$ & $\mathrm{F}$ & 8 & 6 & 26/10 (19) & 4,0 & C & $8-6$ \\
\hline 3 & BMCM & $\mathrm{F}$ & 21 & 11 & 24/7 (17) & 5,4 & C & $10-8$ \\
\hline 4 & ACPJr & $M$ & 36 & 18 & 24/6 (16) & 2,2 & $\mathrm{E}$ & $8-6$ \\
\hline 5 & $J F$ & $\mathrm{~F}$ & 2 & 4 & $55 / 19(36)$ & 2,0 & C & $6-4$ \\
\hline 6 & IGCO & $\mathrm{F}$ & 36 & 13 & 26/8/ (20) & 5,0 & $\mathrm{E}$ & $10-8$ \\
\hline 7 & KRB & $\mathrm{F}$ & 72 & 20 & $50 / 29(39)$ & 6,1 & A & $10-8$ \\
\hline 8 & MAJG & $\mathrm{F}$ & 12 & 10 & $39 / 18(28)$ & 1,4 & $A$ & $6-4$ \\
\hline 9 & ANC & $\mathrm{F}$ & 36 & 13 & $58 / 25(14)$ & 6,0 & A & $10-8$ \\
\hline 10 & IN & $\mathrm{F}$ & 12 & 8 & $32 / 18(27)$ & 4,0 & A & $8-6$ \\
\hline 11 & RAC & $\mathrm{F}$ & 24 & 13 & 21/9 (14) & 4,0 & A & $8-6$ \\
\hline 12 & SFD & $\mathrm{F}$ & 36 & 18 & $60 / 26(48)$ & 3,0 & $\mathrm{D}$ & 8,6 \\
\hline 13 & RRMF & $\mathrm{F}$ & 456 & 80 & $?$ & 4,5 & A & 8,6 \\
\hline 14 & NAS & $\mathrm{F}$ & 5 & 7 & $77 / 41$ (57) & 4,0 & A & $6,4^{*}$ \\
\hline 15 & VSS & $M$ & 108 & 30 & 23/12 (16) & 5,0 & A & $10-8$ \\
\hline 16 & JSQP & $M$ & 42 & 13 & $28 / 16(24)$ & 2,5 & A & $6-4$ \\
\hline 17 & WOB & $M$ & 62 & 22 & 20/14 (18) & 2,9 & A & $8-6$ \\
\hline 18 & MCBR & $\mathrm{F}$ & 84 & 24 & $28 / 12(18)$ & 2,0 & $E$ & $6-4$ \\
\hline 19 & ECBM & $\mathrm{F}$ & 12 & 10 & $35 / 9(21)$ & 3,9 & A & $8-6$ \\
\hline 20 & AVOS & $\mathrm{F}$ & 24 & 15 & $58 / 10(28)$ & 4,0 & A & $8-6$ \\
\hline 21 & MFM & $\mathrm{F}$ & 60 & 22 & $39 / 10(20)$ & 2,0 & A & $6-4$ \\
\hline 22 & JHLO & $M$ & 60 & 30 & $37 / 10(21)$ & 2,0 & $\mathrm{E}$ & $6-4$ \\
\hline 23 & GDT & $\mathrm{F}$ & 18 & 11 & $12 / 4(8)$ & 2,0 & $E$ & $6-4$ \\
\hline 24 & IRC & $\mathrm{F}$ & 264 & 57 & $32 / 12(20)$ & 3,5 & A & $10-8$ \\
\hline 25 & FVA & $\mathrm{F}$ & 4 & 5 & $53 / 12(29)$ & 2,5 & A & $6-4$ \\
\hline 26 & VAM & $\mathrm{F}$ & 32 & 12 & $30 / 18(27)$ & 3,2 & A & $8-6$ \\
\hline 27 & $\mathrm{ABMA}$ & $\mathrm{F}$ & 30 & 10 & 15/6 (11) & 2,5 & A & $6-4$ \\
\hline 28 & JPPT & $M$ & 30 & 17 & $50 / 12(31)$ & 4,5 & A & $10-8$ \\
\hline 29 & TSL & $\mathrm{F}$ & 78 & 22 & $45 / 5$ & 3,5 & A & 8,6 \\
\hline 30 & $\mathrm{TF}$ & $M$ & 36 & 19 & $37 / 12(25)$ & 2,0 & $\mathrm{E}$ & $6-4$ \\
\hline 31 & BRC & $\mathrm{F}$ & 25 & 67 & $36 / 8(20)$ & 2,0 & A & $6-4$ \\
\hline 32 & MLAG & $\mathrm{F}$ & 12 & 9 & $23 / 6(14)$ & 3,0 & A & $8,6^{+}$ \\
\hline 33 & $\mathrm{EBL}$ & $M$ & 24 & 12 & 18/7 (13) & 2,0 & $\mathrm{E}$ & $6-4$ \\
\hline 34 & $\mathrm{RB}$ & $\mathrm{F}$ & 252 & 48 & $21 / 8(12)$ & 2,5 & A & $6-4$ \\
\hline 35 & PRCJr & M & 144 & 49 & $20 / 3(12)$ & 3,5 & A & $8-6$ \\
\hline 36 & CMOL & $\mathrm{F}$ & 36 & 18 & $22 / 10(17)$ & 2,0 & $\mathrm{E}$ & $8-6$ \\
\hline 37 & $\mathrm{ABS}$ & $\mathrm{F}$ & 60 & 17 & $22 / 3(13)$ & 1,0 & $E$ & $5-4$ \\
\hline 38 & RSS & $\mathrm{F}$ & 288 & 52 & $20 / 3(12)$ & 2,0 & A & $8-6$ \\
\hline 39 & JJSS & $M$ & 156 & 55 & 25/12 (18) & 2,5 & A & $8-6$ \\
\hline 40 & GVN & $M$ & 216 & 66 & 20/8 (12) & 4,0 & A & $10-8$ \\
\hline 41 & TLD & $\mathrm{F}$ & 84 & 24 & $40 / 2(22)$ & 6,0 & A & $10-8$ \\
\hline 42 & $\mathrm{ICL}$ & $\mathrm{F}$ & 16 & 12 & $22 / 7$ (16) & 2,0 & A & $8-6$ \\
\hline 43 & MTS & $\mathrm{F}$ & 48 & 17 & 29/15 (23) & 2,0 & $E$ & $6-4$ \\
\hline 44 & JSF & $\mathrm{F}$ & 108 & 32 & $28 / 20(24)$ & 4,0 & A & $8-6$ \\
\hline 45 & GSBV & $\mathrm{F}$ & 60 & 19 & 26/12 (18) & 3,0 & A & $10-8$ \\
\hline 46 & MOCC & $\mathrm{F}$ & 19 & 11 & $38 / 6(27)$ & 3,5 & A & $6-4$ \\
\hline
\end{tabular}

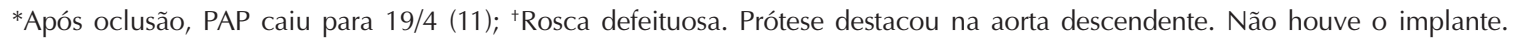


TABELA 1 (continuação)

Caracterização da população do estudo

\begin{tabular}{|c|c|c|c|c|c|c|c|c|}
\hline $\mathbf{N}$ & Id & Sexo & Idade (meses) & Peso (kg) & PAP $(\mathrm{mmHg})$ & $\Phi(\mathbf{m m})$ & Tipo & Prótese \\
\hline 47 & GSP & $\mathrm{F}$ & 12 & 7 & 20/8 (12) & 5,0 & A & $8-6$ \\
\hline 48 & GBG & $M$ & 16 & 12 & 27/12 (16) & 2,0 & A & $6-4$ \\
\hline 49 & MCGS & $\mathrm{F}$ & 96 & 17 & $82 / 58(69)$ & 4,6 & $E$ & $10-8^{\ddagger}$ \\
\hline 50 & DBMO & $M$ & 48 & 17 & 26/12 (14) & 2,0 & A & 6,4 \\
\hline 51 & FCS & $\mathrm{F}$ & 20 & 9 & 44/18 (34) & 3,0 & $A$ & $8-6$ \\
\hline 52 & EPBPN & M & 132 & 40 & 25/8 (15) & 3,0 & $\mathrm{E}$ & $8-6$ \\
\hline 53 & BMTC & $M$ & 23 & 13 & 29/13 (20) & 2,0 & A & $6-4$ \\
\hline 54 & GRL & $M$ & 120 & 20 & 28/5 (16) & 1,0 & $E$ & $6-4$ \\
\hline 55 & SAC & $\mathrm{F}$ & 60 & 18 & $32 / 21(25)$ & 6,0 & $A$ & $10-8$ \\
\hline 56 & FBS & $M$ & 504 & 60 & $22 / 16(18)$ & 8,0 & A & $12-10$ \\
\hline 57 & MEAS & $\mathrm{F}$ & 24 & 18 & $46 / 5(26)$ & 2,0 & A & $6-4$ \\
\hline 58 & GCF & $M$ & 60 & 22 & 28/15 (21) & 0,5 & $\mathrm{E}$ & $6-4$ \\
\hline 59 & PRCV & $\mathrm{F}$ & 18 & 10 & 28/15 (20) & 5,0 & A & $8-6$ \\
\hline 60 & FCS & $\mathrm{F}$ & 72 & 16 & $102 / 64(79)$ & 6,0 & C & $12-10^{\S}$ \\
\hline 61 & PRAN & $M$ & 120 & 33 & $13 / 9(11)$ & 0,5 & $E$ & $6-4$ \\
\hline 62 & MBM & $\mathrm{F}$ & 264 & 52 & $22 / 12(18)$ & 4,0 & C & $10-8$ \\
\hline 63 & $\mathrm{ACL}$ & $\mathrm{F}$ & 48 & 12 & 15/4 (9) & 3,0 & A & $8-6$ \\
\hline 64 & MGSS & $\mathrm{F}$ & 504 & 60 & $32 / 18(23)$ & 4,0 & A & $12-10$ \\
\hline 65 & GBGC & $\mathrm{F}$ & 48 & 12 & 25/10 (19) & 1,0 & $E$ & $6-4$ \\
\hline 66 & LAFA & $\mathrm{F}$ & 24 & 13 & $37 / 13(27)$ & 3,0 & A & $8-6$ \\
\hline 67 & LMD & $M$ & 20 & 11 & $58 / 12(37)$ & 3,0 & $\mathrm{E}$ & $8-6$ \\
\hline 68 & BPS & $M$ & 36 & 20 & 35/11 (23) & 0,6 & A & $6-4$ \\
\hline 69 & EAFP & $\mathrm{F}$ & 60 & 25 & $30 / 14(23)$ & 2,0 & A & $6-4$ \\
\hline 70 & KSB & $\mathrm{F}$ & 96 & 9 & 28/8 (16) & 4,0 & A & $8-6$ \\
\hline 71 & NFNS & $\mathrm{F}$ & 48 & 18 & $30 / 21$ (26) & 2,5 & A & $6-4$ \\
\hline 72 & ACMNC & $\mathrm{F}$ & 6 & 6 & $36 / 15(22)$ & 3,5 & A & $8-6$ \\
\hline 73 & JSE & $\mathrm{F}$ & 20 & 9 & 20/11 (16) & 1,0 & A & $5-4$ \\
\hline 74 & JSA & $\mathrm{F}$ & 72 & 25 & 29/11 (20) & 1,0 & $E$ & 6,4 \\
\hline 75 & PHPS & $M$ & 35 & 12 & $32 / 16(24)$ & 2,0 & A & $8-6$ \\
\hline 76 & PCS & $\mathrm{F}$ & 31 & 54 & $16 / 7(11)$ & 3,5 & A & $10-8$ \\
\hline 77 & MMP & $M$ & 24 & 15 & $45 / 20(31)$ & 1,0 & $\mathrm{E}$ & 5,4 \\
\hline 78 & LVP & $\mathrm{F}$ & 48 & 16 & 40/13 (23) & 2,0 & $\mathrm{E}$ & 6,4 \\
\hline 79 & LMF & $\mathrm{F}$ & 144 & 30 & $29 / 14(21)$ & 4,0 & A & $8-6$ \\
\hline 80 & VL & $\mathrm{F}$ & 96 & 10 & $35 / 19(30)$ & 3,0 & A & $8-6$ \\
\hline 81 & OFMG & $M$ & 216 & 70 & $35 / 12(22)$ & 2,0 & A & $8-6$ \\
\hline 82 & TPRM & $\mathrm{F}$ & 156 & 33 & $41 / 20(28)$ & 3,0 & A & $8-6$ \\
\hline 83 & MFP & $\mathrm{F}$ & 24 & 12 & $29 / 10(21)$ & 2,0 & A & $6-4$ \\
\hline 84 & $\mathrm{LAB}$ & $\mathrm{F}$ & 11 & 10 & $40 / 23(31)$ & 3,5 & $E$ & $8-6$ \\
\hline 85 & JJMC & $M$ & 24 & 9 & 29/15 (21) & 1,0 & $\mathrm{D}$ & $6-4$ \\
\hline 86 & PEOPS & $\mathrm{F}$ & 360 & 76 & $33 / 15(24)$ & 5,0 & A & $10-8$ \\
\hline 87 & PHSM & $M$ & 48 & 17 & $34 / 10(19)$ & 0,5 & $\mathrm{E}$ & $6-4$ \\
\hline 88 & GSV & $M$ & 132 & 33 & $25 / 10(14)$ & 0,5 & $\mathrm{E}$ & $5-4$ \\
\hline 89 & CMJ & $\mathrm{F}$ & 108 & 27 & 23/14 (18) & 0,5 & $\mathrm{E}$ & $6-4$ \\
\hline 90 & SCFA & $\mathrm{F}$ & 600 & 88 & $85 / 20(44)$ & 11 & A & MUSC $12 !$ \\
\hline 91 & LVCA & $\mathrm{F}$ & 36 & 15 & $65 / 35(53)$ & 0,5 & $E$ & $6-4$ \\
\hline
\end{tabular}

${ }^{\ddagger}$ Após oclusão, PAP caiu para 60/32 (55); 'sRealizado na Bahia, em sessão de treinamento; "'HAP grave, sem hiper-resistência. Após oclusão PAP, caiu para 57/15 (31). 


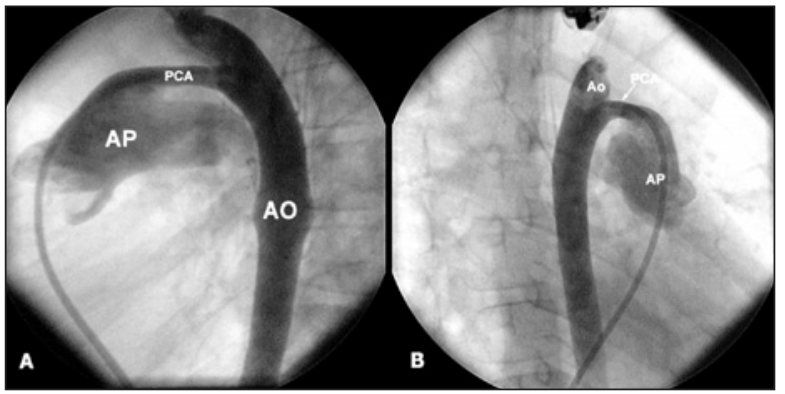

Figura 1 - Angiografia de um canal arterial tubular (Tipo C), medindo 7,6mm. Em A, incidência lateral e, em B, oblíqua anterior direita. Estas incidências permitem a adequada visualização do canal em toda a sua extensão e são ideais para realizar as medidas.

foi superior a $2 \mathrm{~mm}$, foram implantadas próteses Amplatzer. Naqueles menores de $2 \mathrm{~mm}$, foi feita uma tentativa de cruzar, retrogradamente, o canal utilizando um cateter Multipropósito (MPA) ou Judkins para artéria coronária direita (JR). Quando possível, uma ou mais molas destacáveis foram implantadas pelo interior desse cateter, como descrito anteriormente $\mathrm{e}^{10,11}$.
Se, ao contrário, o cateter não conseguisse cruzar a extremidade pulmonar do canal, sua anatomia era avaliada. Na presença de uma ampola aórtica bem formada e corpo com diâmetro e comprimento suficientes para abrigar uma prótese, o paciente era selecionado para o implante de uma ADO.

Com a anatomia favorável, era avançado um guia extra-suporte 0,014 polegadas pelo interior do cateter arterial, posicionando-o profundamente no interior do tronco pulmonar ou ventrículo direito. Sobre ele foi introduzido um cateter-balão $2,0 \mathrm{~mm}$ x $20 \mathrm{~mm}$ de angioplastia coronariana e realizada a dilatação do canal. A seguir, eram feitas tentativas de cruzar, anterogradamente, o ducto com cateter MPA e um guia hidrofílico. Quando possível, a extremidade do cateter era avançada até a aorta descendente, levando no seu interior um guia rígido 0,035 polegadas $/ 260 \mathrm{~cm}$.

Quando não era possível cruzar o canal, o guia rígido era avançado, pelo cateter arterial até o tronco pulmonar, onde era capturado com um laço de 20 ou $25 \mathrm{~mm}$ e retirado pela bainha venosa, criando uma alça artério-venosa (Figura 2).

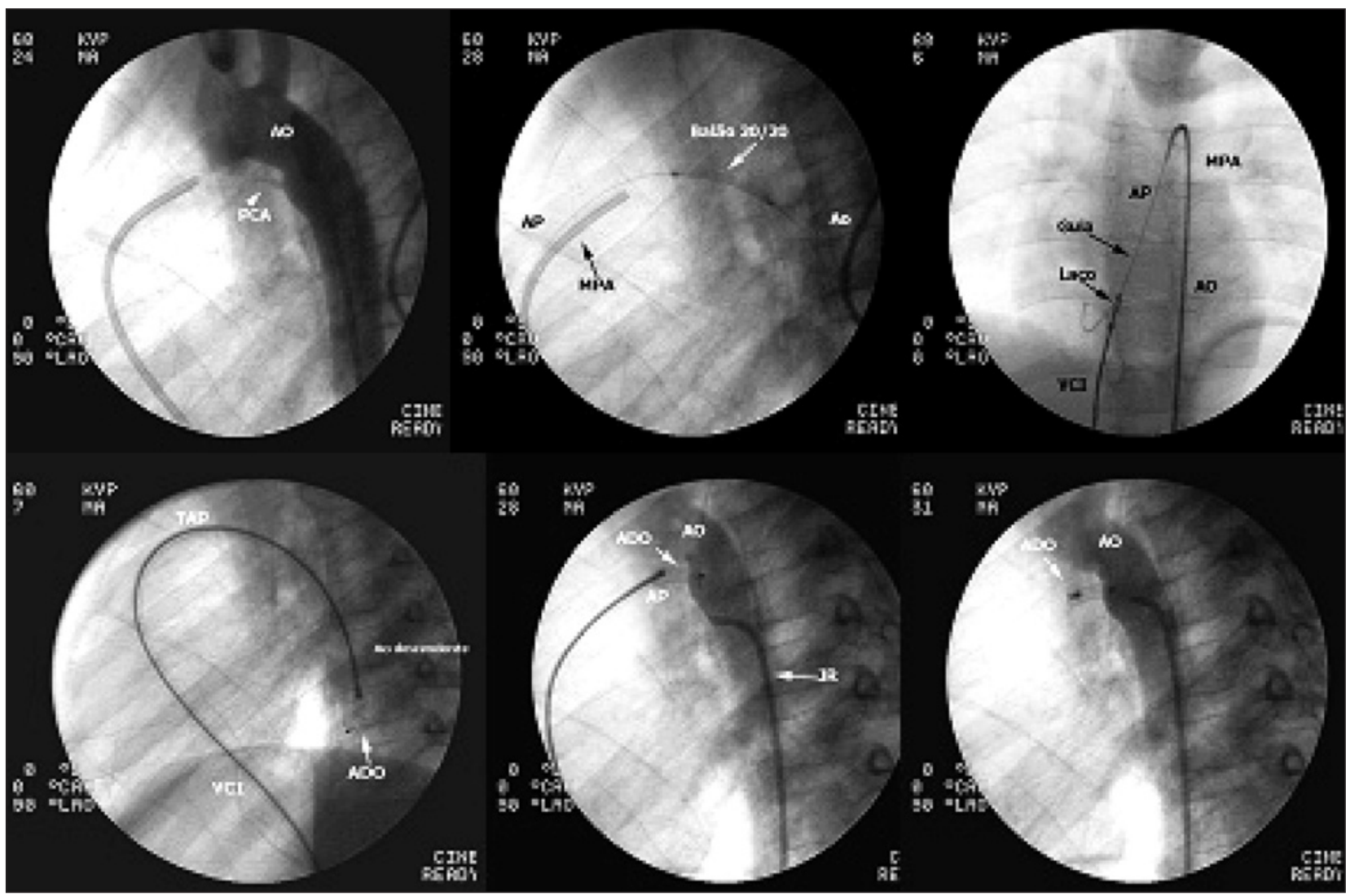

Figura 2 - Detalhes da técnica para fechamento de canal menor do que $2 \mathrm{~mm}$. No painel superior, a figura da esquerda mostra a imagem angiográfica do canal. Note-se que, apesar do pequeno calibre, existe uma ampola aórtica bem formada, capaz de abrigar a prótese. No centro, vê-se a dilatação do canal com balão 2,0 × $20 \mathrm{~mm}$. À direita, o guia rígido é laçado no interior da artéria pulmonar e retirado pela veia cava inferior. No painel inferior, a figura esquerda e a figura central mostram o posicionamento de uma prótese 6-4 no interior do canal arterial. A figura da esquerda mostra o resultado final, com o canal completamente ocluído, sem shunt residual. Não há interferência da prótese com a luz aórtica. 
Com o guia rígido posicionado na aorta descendente, uma bainha longa (AGA Medical Corporation $\left.{ }^{\circledR}\right)$, de calibre compatível com a prótese escolhida, era posicionada na aorta descendente. O guia rígido e o dilatador eram retirados e a prótese, previamente carregada e enroscada no sistema de entrega, era transferida do carregador para a bainha longa e, avançada até a sua extremidade distal. Neste ponto, o disco de retenção era exteriorizado e recuado até se acomodar na ampola ductal.

Estas etapas eram monitoradas por meio de pequenas injeções de contraste pelo cateter arterial, posicionado em frente à ampola aórtica do ducto. Era tomado o cuidado de fazer com que a prótese atravessasse o menor diâmetro do canal de forma a ser comprimida, criando uma dilatação na sua extremidade pulmonar para melhor estabilidade, evitando a embolização do dispositivo para o interior da aorta (Figura 3).

Imediatamente após confirmação do bom posicionamento da prótese, a mesma era destacada do sistema de entrega e realizada nova aortografia descendente, nas mesmas incidências e, repetida após quinze minutos.

O seguimento era feito por meio de ETT antes da alta e, ambulatorialmente, com 1, 3, 6 e 12 meses após o implante.

Os pacientes eram orientados a manter antibioticoterapia profilática por seis meses após o procedimento.

\section{A prótese}

A prótese de canal arterial ADO é um cone truncado formado por malha de Nitinol de 0,004 polegadas, com retalhos de poliéster no seu interior. Ela tem um disco de retenção que fica no interior da ampola aórtica do canal. A porção do cone imediatamente ligada ao disco (diâmetro aórtico) mede $2 \mathrm{~mm}$ a mais do que a sua extremidade (diâmetro pulmonar), onde está localizado o sistema de rosca. Os dispositivos são denominados por dois números que correspondem às medidas dos diâmetros aórtico e pulmonar do cone e vêm em tamanhos de 5-4 a 16-14 mm (Figura 4).

A prótese deve ser escolhida de forma que o diâmetro pulmonar seja, pelo menos, $2 \mathrm{~mm}$ maior do que o menor diâmetro do canal. Portanto, em um canal cujo menor diâmetro fosse $4 \mathrm{~mm}$, deveria ser implantada uma prótese ADO 8-6 mm.

\section{RESULTADOS}

\section{Canais menores de $2 \mathrm{~mm}$}

Do total de 116 pacientes encaminhados para oclusão percutânea, 40 (34\%) tinham o menor diâmetro inferior a $2 \mathrm{~mm}$. Em 25 destes, o canal foi cruzado, retrogradamente, com o cateter MPA e tiveram implantadas molas destacáveis. Os outros 15 foram dilatados e receberam próteses Amplatzer. Destes, 6 (40\%) mediram 0,5 mm; 1 (7\%), 0,6 mm; 7 (46\%), $1 \mathrm{~mm}$ e 1 $(7 \%), 1,4 \mathrm{~mm}$.

Os tipos morfológicos foram do tipo E em 11 $(73 \%)$ casos, ou seja, canais compridos e finos, com importante estreitamento na extremidade pulmonar; 3 (20\%) eram do tipo A, cônico, e 1 (7\%) do tipo D, com múltiplos estreitamentos (Figura 5).

A pressão sistólica na artéria pulmonar foi acima de $30 \mathrm{mmHg}$ em 4 casos, sendo a maior pressão encontrada numa criança com Síndrome de Down (LVCA), que havia sido previamente operada para correção de CIA e CIV ampla.

O implante foi possível em todos os casos. Não houve complicações significativas relacionadas aos procedimentos. Em 6 (40\%) casos, não foi possível cruzar o canal anterogradamente com guia hidrofílico e o guia rígido foi introduzido pelo cateter arterial no interior do tronco pulmonar, de onde foi laçado e retirado pela bainha venosa.

Todos os casos foram encerrados sem shunt resi-

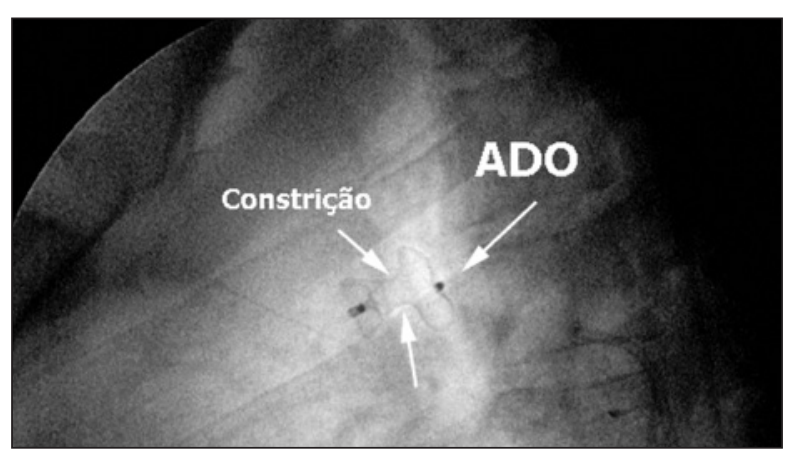

Figura 3 - No detalhe vê-se uma prótese de Amplatzer ductal 10-8 imediatamente após o implante. Observe-se a constrição no meio da prótese. Note-se que a extremidade pulmonar do dispositivo fica maior do que a porção central, como se fosse outro disco de retenção, estabilizando a prótese no interior do canal, desta forma, impedindo sua embolização para a aorta.

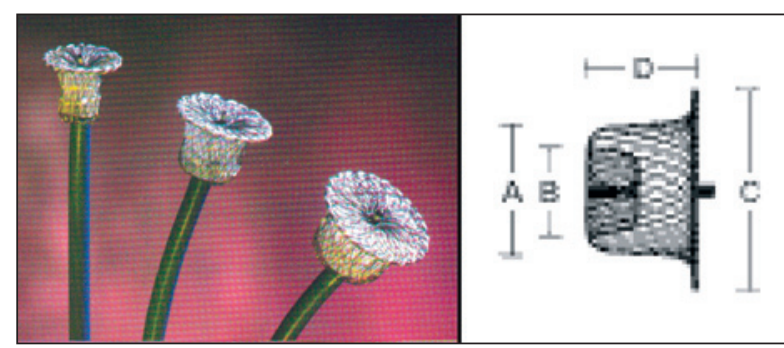

Figura 4 - No painel da esquerda, vêem-se as próteses de Amplatzer Ductal de diferentes tamanhos. No esquema à direita, vê-se, em detalhe, as dimensões da prótese. O diâmetro A (diâmetro aórtico) é $2 \mathrm{~mm}$ maior do que B (diâmetro pulmonar). O disco de retenção C é $4 \mathrm{~mm}$ maior do que A até a prótese 10-8, a partir da qual passa a ser $6 \mathrm{~mm}$ maior. 


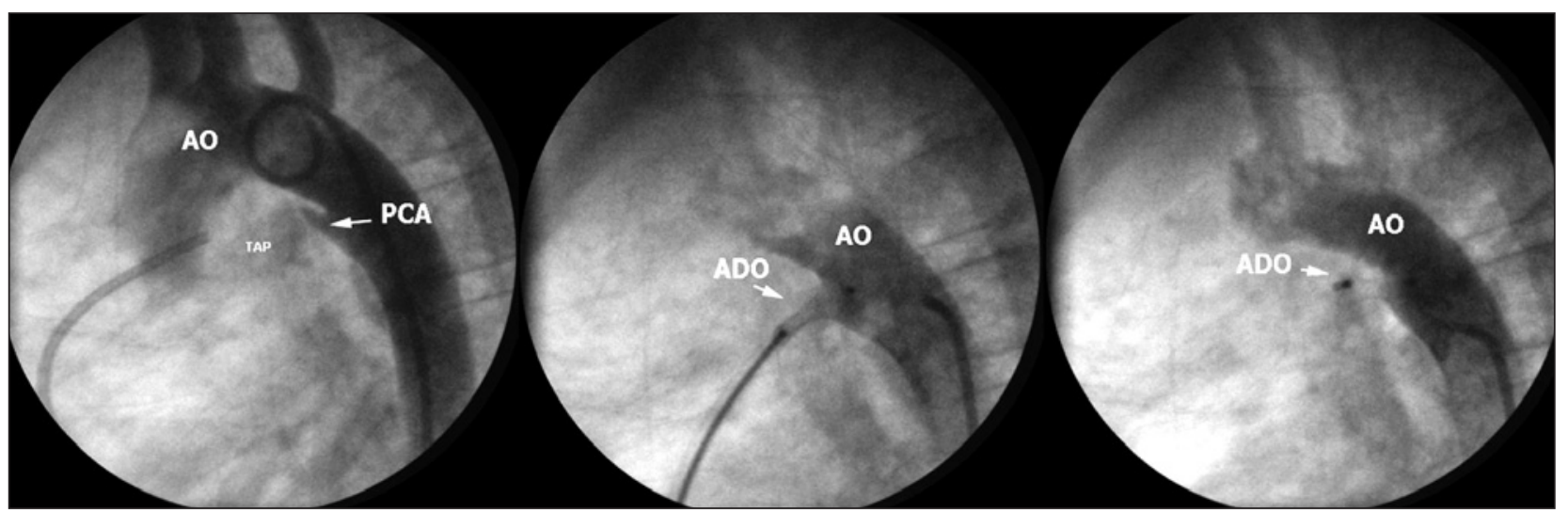

Figura 5 - No painel da esquerda, vê-se um canal arterial tipo E, pequeno, medindo 0,5mm. Apesar das dimensões reduzidas, observe-se a opacificação do tronco pulmonar. No painel central, o canal bastante elástico acomoda bem uma prótese ADO 6-4. À direita, o resultado final após o implante, sem shunt residual. Observe-se o dispositivo bem adaptado à anatomia do canal, sem qualquer interferência com a luz aórtica.

dual detectado pela angiografia de controle. Os ecocardiogramas de seguimento também não demonstraram fluxo residual, nem obstruções ao fluxo em aorta descendente ou ramo esquerdo pulmonar.

\section{Canais maiores de $2 \mathrm{~mm}$}

Os demais 76 casos tinham canais maiores do que $2 \mathrm{~mm}$, cujos diâmetros variaram entre 2 e $11 \mathrm{~mm}$ $(3,53 \pm 1,64 \mathrm{~mm})$. Foram do tipo A em 55 (72\%) casos, tipo C em 6 (8\%), tipo D em 1 (2\%) e tipo E em $14(18 \%)$.

As pressões pulmonares foram acima de $30 \mathrm{mmHg}$ em $36(47 \%)$ casos e, acima de $70 \mathrm{mmHg}$ em 4 (5\%). Nestes últimos, foram avaliadas as resistências vasculares. Não houve hiper-resistência vascular pulmonar fixa e notou-se queda da pressão arterial pulmonar, em todos os 4 casos, após o fechamento do canal (Tabela 1).

Em 75 casos, foram utilizadas ADO, sem maiores dificuldades. No caso (SCFA) de uma paciente de 50 anos, foi utilizada uma prótese de VSD MUSC, como preconizado por Thanopoulos et al. ${ }^{12}$. Neste caso, também, foi realizada a medida do diâmetro estirado do canal com um balão medidor AGA de 24 mm (Figura 6).

O implante foi possível em todos os casos, exceto um (MLAG), em que uma rosca defeituosa causou o destacamento inadvertido de uma ADO 8-6 na aorta descendente, onde se alojou na altura da artéria mesentérica superior. Não houve interrupção de fluxo para esse vaso ou para a aorta distal à prótese. Embora não houvesse cateter laço disponível, foi improvisado um laço com guia de angioplastia. Foram feitas várias tentativas, mal-sucedidas, de resgate e, por fim, a prótese foi conduzida, por meio de um guia rígido no interior de um dilatador de bainha de Mullins, até a altura da ampola aórtica do canal, onde foi estabilizada. A paciente foi encaminhada para cirurgia, onde teve a prótese retirada e o canal arterial ligado sem maiores complicações.
Em um caso, ocorreu diminuição transitória do pulso arterial, no membro puncionado, que cedeu nas primeiras horas após o procedimento.

A paciente SCFA de 50 anos e portadora de um canal medindo $11 \mathrm{~mm}$ vinha sendo tratada para fibrilação atrial crônica antes do cateterismo e apresentou fibrilação atrial com baixa resposta ventricular na indução anestésica. Na reversão da anestesia, teve quadro de baixo débito, não respondendo à medicação instituída. Foi implantado marca-passo provisório para estabilização do quadro clínico. Posteriormente, a equipe de eletrofisiologia indicou marca-passo definitivo, que foi implantado no $4^{\circ}$ dia de pós-operatório. A paciente teve alta hospitalar com o canal fechado e em boas condições hemodinâmicas.

Dez pacientes apresentaram alguma passagem de fluxo pelo interior da prótese, na angiografia de controle imediato, mas sem jato. O fluxo persistiu no eco realizado na primeira semana após o implante, em dois pacientes (Figura 7), tendo desaparecido em todos após um mês. Mantêm-se completamente fechados, até o presente momento, sem fluxo residual.

Não houve alteração significativa de fluxo em ramo esquerdo pulmonar ou na aorta descendente em nenhum dos casos.

Não houve complicações tardias em nenhum dos grupos.

\section{DISCUSSÃO}

Há vantagens no fechamento percutâneo dos canais arteriais quando comparados ao fechamento cirúrgico. Maior conforto para o paciente, menos dor, menor tempo de internação, ausência de cicatriz e eficácia comparável à cirúrgica tornam a oclusão percutânea o método terapêutico de escolha, na maioria dos serviços em todo o mundo. 


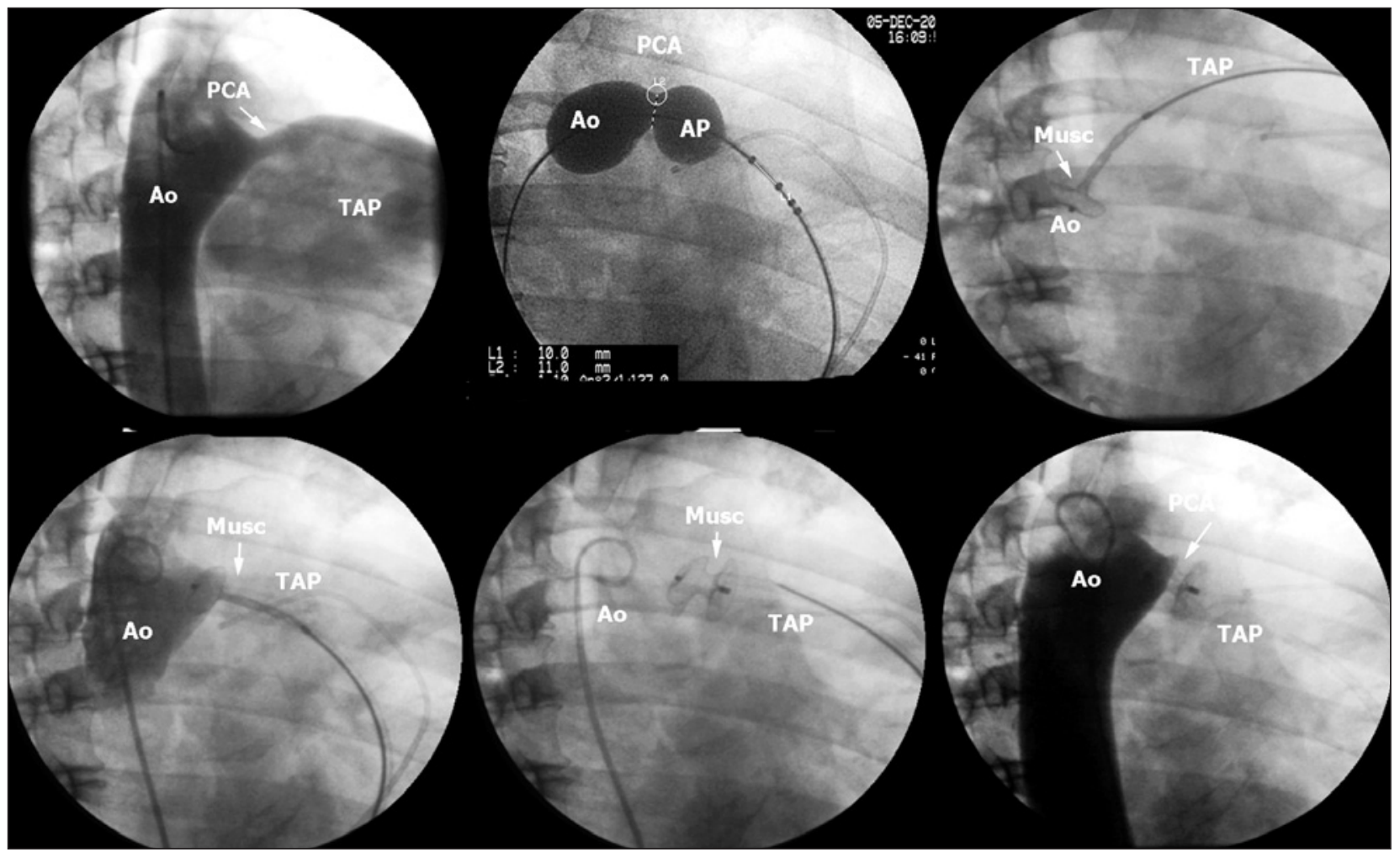

Figura 6 - Caso no 90 (SCFA). No painel superior: à esquerda, a imagem angiográfica do canal em OAD. No centro, a medida do diâmetro estirado do canal utilizando-se um balão medidor AGA de $24 \mathrm{~mm}$. À direita, a abertura do disco distal de uma prótese de CIV muscular de $12 \mathrm{~mm}$. No painel inferior: à esquerda, o posicionamento do dispositivo no interior do canal visualizado por meio de injeção manual de contraste pelo cateter arterial. No centro, a prótese liberada. À direita, aortografia descendente de controle em OAD mostrando o canal completamente fechado, sem shunt residual.

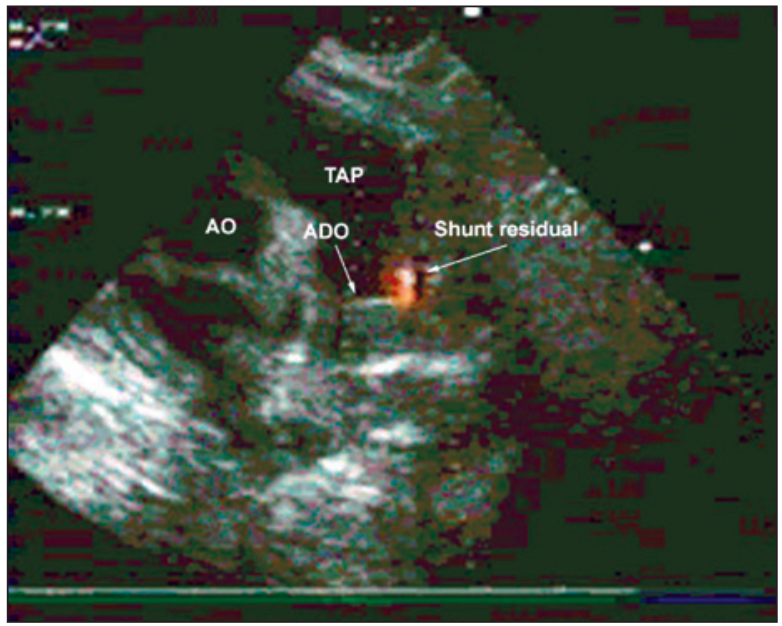

Figura 7 - Ecocardiograma com fluxo a cores realizado no dia seguinte ao procedimento. Note-se o padrão de fluxo residual de pequeno alcance, totalmente laminar. Este fluxo não estava presente no ecocardiograma realizado na semana seguinte.

Vários dispositivos vêm sendo utilizados para o fechamento dos canais, sendo os mais empregados para esse fim as molas de Gianturco, as de liberação controlada $^{13-16}$ e, a prótese Amplatzer ${ }^{17,18}$. Mais recen- temente, um novo tipo de mola pré-moldada em cone duplo (Nit Occlud PFM ${ }^{\circledR}$ ), também tem sido utilizado ${ }^{19}$.

Complicações com o uso das próteses Amplatzer são raras e só ocorrem precocemente. Não há, até o momento, relatos de complicações tardias com o uso destes dispositivos. Obstruções de ramo esquerdo pulmonar ou aorta descendente são excessivamente ra$\operatorname{ras}^{2,4,7}$. Foi relatado apenas um caso de morte em que a prótese embolizou para aorta descendente ${ }^{17}$.

A maior desvantagem do uso das próteses Amplatzer é, indubitavelmente, o custo, bem maior quando comparado aos dos outros oclusores disponíveis no mercado.

\section{Canais maiores do que $2 \mathrm{~mm}$}

O procedimento é bastante simples, nesses casos. Algum cuidado deve ser tomado no posicionamento da bainha longa na aorta descendente, principalmente em crianças pequenas. As bainhas longas da AGA são muito pouco resistentes e, mesmo com o dilatador no seu interior, praticamente invisíveis à fluoroscopia. Por vezes, o medo de não haver cruzado o canal faz com que ela seja introduzida muito distalmente no interior da aorta descendente, tornando excessivamente acentuada a curva ao atravessar a via de saída do ventrículo 
direito e artéria pulmonar. Quando o guia e dilatador são retirados, pode-se produzir uma dobradura (kinking), impedindo a progressão da prótese. Geralmente, o recuo da bainha para perto da extremidade aórtica do canal torna a curva mais suave, e evita essa complicação. O uso de bainhas mais flexíveis (por exemplo, aramadas) pode ser necessário em alguns casos. De toda forma, seria extremamente desejável que a extremidade distal da bainha tivesse uma marca radiopaca, o que facilitaria sobremaneira o seu correto posicionamento.

Os canais hipertensos merecem algum cuidado, sendo fundamental o cálculo de fluxos e resistências para a indicação do fechamento. A alta pressão na artéria pulmonar faz com que aumente o risco de embolização do dispositivo para a aorta descendente. Nestes casos, como as ADO não têm disco do lado pulmonar, deverão ser superdimensionadas para impedir seu deslocamento após o implante. O grande diâmetro de alguns desses canais impossibilita o uso das ADO e torna necessário o uso das próteses de VSD MUSC, que apresentam um disco em cada extremidade. $\mathrm{O}$ procedimento com elas é exatamente igual ao das ADO e não acarreta nenhuma dificuldade adicional ${ }^{12}$ (Figura 6). Canais de calibre muito grande podem ser ocluídos com outros tipos de prótese Amplatzer, como as de comunicação interatrial (Amplatzer Septal Occluder- ASO), por exemplo ${ }^{20}$.

Nos canais muito grandes, nos que tenham orientação espacial diferente do habitual ou nos pacientes obesos, a dificuldade na obtenção de uma imagem angiográfica adequada pode impedir a medida acurada dos diâmetros para a apropriada escolha da prótese e causar a embolização do oclusor. Para os casos mais difíceis, a mensuração do diâmetro estirado do canal com balões medidores pode resolver o problema. Além da medida mais correta, o balão pode demonstrar melhor a anatomia do defeito ${ }^{12}$ (Figura 6).

Outra causa de preocupação são os grandes canais em crianças muito novas, com baixo peso $^{5,6}$. Na maioria das vezes, quando se implantam grandes oclusores, em função do pouco espaço no istmo aórtico, o bordo superior do disco de retenção pode invadir a luz aórtica e provocar obstruções indesejadas, determinando a retirada do dispositivo e, inviabilizando o procedimento com os modelos de próteses habituais. Para superar esse inconveniente, já existem, disponíveis, próteses anguladas e assimétricas, com o bordo superior do disco de retenção menor do que o inferior. Desta forma, elas preenchem a ampola aórtica, sem interferir com a sua luz ${ }^{21}$.

\section{Canais menores do que $2 \mathrm{~mm}$}

É quase que unanimemente aceito que canais cujo menor diâmetro seja inferior a $2 \mathrm{~mm}$ são adequados ao fechamento com molas ${ }^{19}$. Considera-se um procedimento fácil, de baixo risco e alta efetividade, uma vez que as molas ocupariam praticamente toda a luz do canal, ocluindo-o com segurança. Por outro lado, mesmo em canais angiograficamente bastante pequenos, uma segunda mola pode ser necessária por apresentarem shunts residuais dentro do primeiro ano de seguimento. Isto ocorre por recanalização de um ducto anteriormente fechado, pois, devido ao processo de fibrose resultante do fechamento, a mola pode se retrair e se desalojar o suficiente para criar novo pertuito acima dela.

Outra situação que ocorre, algumas vezes, é a manutenção de um pequeno shunt residual que, na avaliação durante o procedimento, "certamente vai se fechar nos próximos dias/meses" e é deixado aberto. Às vezes, não se fecha espontaneamente, e necessita do implante de nova mola. Isto é evitado com a política atual de não se permitir qualquer shunt residual, implantando tantas molas quantas necessárias para obter a oclusão completa do defeito, ainda no laboratório de hemodinâmica ${ }^{8,22}$.

É importante, no entanto, que se ressaltem alguns aspectos desse procedimento: um dado que tem sido, a nosso ver, muito negligenciado é que as estruturas orgânicas têm elasticidade e são, portanto, passíveis de se distender. Temos tido oportunidade de nos deparar com alguns canais angiograficamente muito pequenos, mas que apesar disso têm diâmetro e comprimento perfeitamente capazes de alojar as ADO (Figura 5).

Por outro lado, alguns desses canais apresentam importante estenose na sua extremidade pulmonar, por vezes, impossibilitando cruzá-los retrogradamente para a liberação das molas. Neste caso, para ultrapassar este obstáculo com o cateter se faz necessária a sua dilatação e, mesmo, a captura do guia com laço na artéria pulmonar, para fornecer mais suporte ao avanço do cateter. Isso adiciona maior complexidade ao processo, mesmo para a colocação retrógrada de uma mola, e torna o implante de uma ADO não mais complicado.

Em função do acima exposto, considerando que as próteses Amplatzer têm uma taxa de oclusão mais alta (98-100\% versus 72,3-96,7\%), e menor índice de complicações do que as molas ${ }^{2-5,7,13-16}$, modificamos nossa estratégia. Ao lidar com um canal angiograficamente pequeno, não sendo possível cruzá-lo retrogradamente, procedemos à sua dilatação com cateterbalão de angioplastia coronariana. Se a anatomia do canal for compatível, a prótese Amplatzer será nossa melhor escolha.

\section{CONCLUSÕES}

O uso das próteses Amplatzer é fácil, extremamente seguro, com baixa morbidade e altíssima eficácia.

O uso do oclusor Amplatzer é possível mesmo em canais de muito pequeno calibre, com excelente resultado, sem adicionar muita dificuldade ao procedimento. 
Achamos que as próteses Amplatzer devam ser a primeira opção para a oclusão percutânea dos canais arteriais de qualquer calibre com anatomia compatível, desde que o custo do procedimento não seja um impedimento.

\section{REFERÊNCIAS BIBLIOGRÁFICAS}

1. Latson LA. Residual shunts after transcatheter closure of patent ductus arteriosus. A major concern or benign "technomalady"? Circulation 1991;84:2591-3.

2. Masura J, Walsh KP, Thanopoulos B, Chan C, Bass J, Goussous $Y$, et al. Catheter closure of moderate-to-large size patent ductus arteriosus using the new Amplatzer duct occluder: immediate and short-term results. J Am Coll Cardiol 1998;31:878-82.

3. Butera G, de Rosa G, Chessa M, Piazza L, Delogu A, Frigiola A, et al. Transcatheter closure of persistent ductus arteriosus with the Amplatzer duct occluder in very young symptomatic children. Heart 2004;90:1467-70.

4. Pass RH, Hijazi ZH, Hsu DT, Lewis V, Hellenbrand WE. Multicenter USA Amplatzer patent ductus arteriosus device trial: initial and one-year results. J Am Coll Cardiol 2004; 44:513-9.

5. Al-Ata J, Arfi AM, Hussain A, Kouatli AA, Jalal MO. The efficacy and safety of the Amplatzer ductal occluder in young children and infants. Cardiol Young 2005;15:279-85.

6. Jan SL, Hwang B, Fu YC, Chi CS. Transcatheter closure of a large patent ductus arteriosus in a young child using the Amplatzer duct occluder. Pediatr Cardiol 2005;26:703-6.

7. Masura J, Tittel P, Gavora P, Podnar T. Long-term outcome of transcatheter patent ductus arteriosus closure using Amplatzer duct occluders. Am Heart J 2006;151:755.

8. Wang JK, Hwang JJ, Chiang FT, Wu MH, Lin MT, Lee W$\mathrm{L}$, et al. A strategic approach to transcatheter closure of patent ductus: Gianturco coils for small-to-moderate ductus and Amplatzer duct occluder for large ductus. Int J Cardiol 2006;106:10-5.

9. Krichenko A, Benson LN, Burrows P, Möes CA, McLaughlin $\mathrm{P}$, Freedom RM. Angiographic classification of the isolated, persistently patent ductus arteriosus and implications for percutaneous catheter occlusion. Am J Cardiol 1989;63:877-80.

10. Tometzki AJ, Arnold R, Peart I, Sreeram N, Abdulhamed JM, Godman MJ, et al. Transcatheter occlusion of the patent ductus arteriosus with Cook detachable coils. Heart 1996; 76:531-5.
11. Hazama K, Nakaninshi T, Tsuji T, Kinugawa Y, Matsuoka $\mathrm{S}$, Mori K, et al. Transcatheter occlusion of arterial duct with new detachable coils. Cardiol Young 1996;6:332-6.

12. Thanopoulos BD, Tsaousis GS, Djukic M, Al Hakim F, Elefthterakis NG, Simeunovic SD. Transcatheter closure of high pulmonary artery pressure persistent ductus arteriosus with the Amplatzer muscular ventricular septal defect occluder. Heart 2002;87:260-3.

13. Parra-Bravo JR, Acosta-Valdez JL, Giron-Vargas AL, BeiranaPalencia LG, Rodriguez-Hernandez L, Estrada-Loza M de J, et al. Transcatheter occlusion of the patent ductus arteriosus with detachable coils: immediate results and intermediate-term follow-up. Arch Cardiol Mex 2005;75:413-20.

14. Brown S, Bruwer A, Al-Zaghal A, Claassens A. Effectiveness of single detachable Cook coils in closure of the patent ductus arteriosus. Cardiovasc J S Afr 2004;15:76-80.

15. Mahayni MN, Pepin-Donat M, Saillant D, Poinsot J, Vaillant MC, Chantepie A. Patent ductal arteriosus occlusion by Rashkind umbrella and by detachable coil. Arch Mal Coeur Vaiss 2002;95:418-24.

16. Galal MO, Bulbul Z, Kakadekar A, Fatani AE, de Moor M, el-Oufi $S$, et al. Comparison between the safety profile and clinical results of the Cook detachable and Gianturco coils for transcatheter closure of patent ductus arteriosus in 272 patients. J Interv Cardiol 2001;14:169-77.

17. Faella HJ, Hijazi ZM. Closure of the patent ductus arteriosus with the Amplatzer PDA device: immediate results of the international clinical trial. Catheter Cardiovasc Interv 2000;51:50-4.

18. Bilkiss AA, Alwi M, Hasri S, Haifa AL, Geetha K, Rehman MA, et al. The Amplatzer duct occluder: experience in 209 patients. J Am Coll Cardiol 2001;37:258-61.

19. Celiker A, Aypar E, Karagoz T, Dilber E, Ceviz N. Transcatheter closure of patent ductus arteriosus with Nit-Occlud coils. Catheter Cardiovasc Interv 2005;65:569-76.

20. Spies C, Ujivari F, Schräder R. Transcatheter closure of a $22 \mathrm{~mm}$ patent ductus arteriosus with an Amplatzer atrial septal occluder. Catheter Cardiovasc Interv 2005;64:352-5.

21. Vijayalakshmi IB, Chitra N, Rajasri R, Prabhudeva AN. Amplatzer angled duct occluder for closure of patent ductus arteriosus larger than aorta in an infant. Pediatr Cardiol 2005; 26:480-3.

22. Zellers TM, Wylie KD, Moake L. Transcatheter coil occlusion of the small patent ductus arteriosus $(<4 \mathrm{~mm})$ : improved results with a "multiple coil - no residual shunt" strategy. Catheter Cardiovasc Interv 2000;49:307-13. 